Apuntes Universitarios, 2018: 8(1), enero-abril, págs. ...

ISSN: ..., doi:

\title{
Revisión teórica sobre el perfil cognitivo del perfeccionismo desadaptativo
}

\author{
Somia Chemisquy ${ }^{1 \mathrm{a}}$ \\ Universidad Nacional de Córdova, Argentina ${ }^{1}$ \\ Orcid ID: https://orcid.org/0000-0002-3820-3036¹
}

Recibido: 13 de setiembre de 2017

Aceptado: 13 de diciembre de 2017

\begin{abstract}
Resumen
El perfeccionismo puede ser entendido como la tendencia a establecer estándares de desempeño elevados y hasta irracionales, a realizar una autoevaluación estricta y rígida, y a medir el valor personal en relación a los logros y/o fracasos. El modelo cognitivo propone la descripción de perfiles cognitivos en los que se incluyen algunos componentes principales como los esquemas, las creencias, las distorsiones cognitivas, los pensamientos automáticos y el afrontamiento. En este trabajo se procuró describir el perfil cognitivo de los perfeccionistas desadaptativos para lo que se realizó una revisión no sistemática de la bibliografía buscando evidencias empíricas de los componentes de ese perfil. Se hallaron evidencias sobre la presencia de esquemas principalmente referidos al dominio de desconexión/rechazo, creencias acerca de la necesidad perfección y la auto-valoración contingente, un sesgo interpretativo de perfección y otros como la atención selectiva y el pensamiento dicotómico, diversos pensamientos automáticos de tipo perfeccionista que se suelen asociar a pensamientos negativos más generales y que perseveran en forma de rumiaciones y autocrítica; y un estilo de afrontamiento enfocado en la emoción que no logra neutralizar las situaciones estresantes. Los lectores encontrar en este artículo información accesible y actualizada útil para identificar objetivos preventivos o de tratamiento.
\end{abstract}

Palabras clave: perfeccionismo desadaptativo - esquemas - creencias - afrontamiento cogniciones

\section{Theoretical review on maladaptive perfectionism's cognitive profile}

\begin{abstract}
a Correspondencia al autor

E-mail: sochemisquy@gmail.com
\end{abstract}

Perfectionism may be understood as the tendency to set high and irrational standards, to stringently self evaluate and to asses self-worth based on achievement or failure. The cognitive 
model proposes the identification of cognitive profiles comprised of: schemas, beliefs, cognitive distortions, automatic thoughts and coping. The aim of this work was to describe maladaptive perfectionist's cognitive profile. A non-systematic scientific literature review was made and empiric evidence was found. Maladaptive perfectionist's cognitive profile was described as the presence of schemas belonging to the disconnection/reject domain; beliefs about one's need to be perfect and beliefs about contingent self-worth; biased interpretation of perfection, attentional bias and dichotomous thinking; perfectionistic automatic thoughts that associate with general negative cognitions, which tend to be maintained through rumination and self-criticism; and an emotion-focused coping style which is unhelpful to neutralize stressful situations. This article brings accessible and updated information which can be, useful to recognize the maladaptive perfectionist's profile, and to identify goals for prevention or treatment interventions.

Key-words: maladaptive perfectionism - schemas - beliefs - coping - cognition

\section{Introducción}

El perfeccionismo puede ser entendido como la tendencia a establecer estándares de desempeño elevados y hasta irracionales, a realizar una autoevaluación estricta y rígida, y a medir el valor personal en relación a los logros y/o fracasos (Hewitt \& Flett, 1991).

Actualmente la mayoría de los investigadores están de acuerdo en que el perfeccionismo es multidimensional y que sus distintas manifestaciones pueden ser subsumidas en dos factores de orden superior: las preocupaciones perfeccionistas, y los esfuerzos perfeccionistas (Bieling, Israeli, \& Antony, 2004). Las primeras representan el aspecto más negativo o insano del perfeccionismo, y comprenden el perfeccionismo socialmente prescrito, las preocupaciones por cometer errores, las excesivas demandas parentales, la autocrítica exagerada, y la discrepancia percibida entre los estándares auto-impuestos y los logros (Stoeber \& Otto, 2006). Se las considera desadaptativas porque las investigaciones han demostrado su impacto negativo en la salud física y mental de las personas, llevando al desarrollo de problemas tales como fatiga (Magnusson, Nias \& White, 1996), trastornos de la conducta alimentaria (Bardone-Cone et al., 2007), depresión (Cha, 2016), sintomatología obsesivo-compulsiva (Ashby \& Bruner, 2005), entre otros. Por su parte, los esfuerzos perfeccionistas representan un aspecto más saludable o positivo, que incluye al perfeccionismo autorientado y que se explica como el afán por alcanzar las metas, y la búsqueda de la excelencia. Aún no queda claro el impacto de este perfeccionismo adaptativo en la salud mental: algunos estudios verifican que, cuando se controlan las preocupaciones perfeccionistas, este perfeccionismo adaptativo se asocia a características más bien positivas; mientras que otros estudios continúan asociando esta dimensión a vulnerabilidad psicológica, en especial para trastornos de la conducta alimentaria (Bardone-Cone et al., 2007) 
y a mayor reactividad ante el estrés (Flett, Nepon, Hewitt, \& Fitzgerald, 2016; Hewitt \& Flett, 1993).

El modelo de dos componentes de orden superior (i.e. preocupaciones y esfuerzos perfeccionistas) se completa con el perfeccionismo orientado a los otros que, al igual que el autorientado, comprende las exigencias de perfección, pero en este caso destinadas a los otros significativos (Sirois \& Molnar, 2015). Este tipo de perfeccionismo, que fue descrito inicialmente por Hewitt y Flett (1991), reveló en las investigaciones sus relaciones diferenciadas con resultados psicológicos distintos a las otras dimensiones: este tipo de perfeccionismo está más relacionado a características propias de personalidades narcisistas y/o antisociales (Sherry, Gralnick, Hewitt, Sherry, \& Flett, 2014; Stoeber, 2014; 2015).

Cuando el perfeccionismo es disfuncional, sus consecuencias negativas no sólo implican la posibilidad de desarrollar algún trastorno mental. Este tipo de perfeccionismo también deteriora la vida cotidiana de las personas debido a sus correlatos conductuales y sociales. Entre los primeros se encuentran la tendencia a la comprobación, la comparación con otros (que tiende a ser hacia arriba, es decir con otros mejores a él), la búsqueda de reaseguro de que han hecho las cosas bien por medio de interminables preguntas a los demás, y otros comportamientos contraproducentes, que sirven al perfeccionista para descender la ansiedad y que pueden tomar distintas formas (p.ej. armado de listas, excesiva planificación) (Egan, Wade, Shafran \& Antony, 2014).

Por su parte, la vida social de los perfeccionistas puede verse afectada por la presencia de un estilo de relación caracterizado por la presencia hostilidad y sensibilidad interpersonal: estos individuos pueden ser hoscos y agresivos en el trato a las personas, mientras que en su fuero interno son muy sensibles a lo que dicen y hacen los demás (Hewitt, Fleet, Sherry \& Caelian, 2006). Esta sensibilidad interpersonal se expresa como desesperanza en las relaciones sociales (Roxborough et al., 2012) y como ansiedad social (Goya Arce \& Polo, 2016), y lleva a los perfeccionistas a interpretar de manera distorsionada las relaciones sociales y sus resultados.

La presencia de hostilidad y sensibilidad interpersonal puede llevar a la experimentación de conflictos interpersonales (Hill, Zrull \& Turlington, 1997) y de pareja (Furman, Luo, \& Pond, 2017), y a la desconexión social objetiva y subjetiva. Mientras que objetivamente pueden sentirse desconectados al ser rechazados por los demás, se sienten desconectados subjetivamente porque perciben que cuentan con escaso apoyo y contención de parte de sus redes sociales (Sherry, Law, Hewitt, Flett, \& Besser, 2008), porque se sienten solos (Goya Arce \& Polo, 2016), y porque sienten que no son importantes para los demás (Flett, GalfiPechenkov, Molnar, Hewitt, \& Goldstein, 2012; Cha, 2016). 
Desde el marco teórico cognitivo-conductal se acepta que las personas tienen un determinado perfil cognitivo que define su manera particular de ser y estar en el mundo. Este perfil se describe en función al estilo de procesamiento de la información con el que las personas captan los estímulos provenientes del medio y los interpretan, dando lugar a las respuestas comportamentales y emocionales (Beck, et al., 1995). Generalmente se incluyen algunos componentes principales al analizar un perfil cognitivo, entre ellos: los esquemas, las creencias, las distorsiones cognitivas y el afrontamiento (Lemos Hoyos, Jaller Jaramillo, González Calle, Díaz León \& De La Ossa, 2012; Álvarez, Bedoya, \& Arredondo, 2010).

Los esquemas son las estructuras que organizan y dan significado a la experiencia, estableciendo algo así como las coordenadas a partir de las cuales se va a interpretar el mundo, a los otros y a uno mismo. Es importante destacar que los esquemas tienen un componente motivacional además del ideacional, ya que mueven a la persona a accionar en el mundo en virtud de la interpretación resultante del procesamiento de la información (Keegan, 2007). Algunos de estos esquemas se desarrollan muy temprano en la vida y pueden ser desadaptativos, en el sentido de que organizan la experiencia en base a un sistema de interpretaciones autoderrotistas que llevan a las personas a responder con comportamientos más bien disfuncionales. Pueden describirse 18 esquemas maladaptativos tempranos (p.ej. abandono/inestabilidad, autosacrificio, estándares implacables/hipercriticismo, fracaso) que se organizan en dominios más amplios (p.ej. desconexión/rechazo, focalización en los otros, límites insuficientes). Estos dominios se conforman a partir de las necesidades básicas del desarrollo humano que pueden resultar insatisfechas, o al contrario exageradamente repletas, durante el crecimiento (Young, Klosko, \& Weishaar, 2003).

Los esquemas se despliegan como una manera de explicar los pensamientos, conductas y emociones que aparecen en el intercambio con el mundo (Beck, 2000). De este modo, luego de una crianza que ha dejado un dominio de necesidades insatisfechas, el esquema puede dar sentido a la experiencia pasada, actual y la por venir. Estos esquemas tienden a perpetuarse utilizando mecanismos como la distorsión cognitiva, y los estilos de afrontamiento (Young et al., 2003).

Gracias a las distorsiones cognitivas se mantiene la coherencia interna por medio de un procesamiento sesgado de la información, de manera que el individuo puede, por ejemplo, atender selectivamente a los errores que comete, y dejar pasar las experiencias de éxito en orden a mantener, por ejemplo la idea: "soy un fracasado". Se entiende que estos sesgos cognitivos no modifican la realidad del mundo externo, sino que funcionan como unas gafas de color que tiñen la visión e interpretación del mundo que hace la persona. Algunos de estos sesgos son: la 
catastrofización, el pensamiento dicotómico todo o nada, la inferencia arbitraria, la lectura de mente y la minimización (Yurica \& DiTomasso, 2005).

Los esquemas y las distorsiones cognitivas representan los procesos cognitivos, a diferencia de las creencias y los pensamientos automáticos que son los contenidos resultantes de esos procesos.

Las creencias son aquellas ideas que la persona tiene acerca de sí misma, el mundo y los demás. Aunque no necesariamente tengan entidad consciente, son verdades absolutas para la persona, que cree en ellas, aunque su estatus epistemológico sea dudoso (Beck, 2000; Pascal, 2003). Algunas creencias se organizan como reglas o normas de comportamiento y habitualmente se las conoce como creencias intermedias, para diferenciarlas de las nucleares que versan sobre aspectos amplios del sí mismo, la realidad y los otros. Las creencias intermedias suelen enunciarse en términos de "si... entonces..." (Keegan, 2007). En el ejemplo ya mencionado se puede pensar que "soy un fracasado" sería una creencia nuclear, de la cual puede derivar una regla o creencia intermedia como "como soy un fracasado, entonces debo hacer todo perfecto para que los demás me quieran".

Otro tipo de producto cognitivo son aquellas ideas o imágenes que aparecen en la consciencia de la persona cuando se expone a ciertos estímulos, y que desencadenan una respuesta emocional y/o conductual. Se denominan pensamientos automáticos debido a su aparición inesperada, y a la habitual dificultad de controlarlos, cuyo aprendizaje es uno de los objetivos de la terapia cognitiva estándar (Vázquez \& Cameron, 1997).

El último elemento que se especifica en un perfil cognitivo es el estilo de afrontamiento. Con este concepto se hace referencia a las estrategias que las personas utilizan para sobrellevar las situaciones de estrés a las que se enfrentan, ya sean eventos estresantes o ajetreos diarios. El afrontamiento es un proceso en cuyo primer paso se lleva a cabo una evaluación en dos partes: en la primera de ellas se evalúa el riesgo implicado en la situación a afrontar, y en la segunda se analiza con qué recursos se cuenta y qué estrategia es la más apropiada para afrontarla (Lazarus \& Folkman, 1987). Es evidente que al momento de realizar esas evaluaciones entran en juego los esquemas y los sesgos, que van a imprimir un modo particular de interpretar y, por ende, de evaluar los estresores y las estrategias de afrontamiento a utilizar.

Si bien el perfeccionismo es un tema que que ha sido estudiado con diversos objetivos, hasta el momento no se encontraron artículos en habla hispana que resuman los hallazgos de esos estudios describiendo el perfil cognitivo de los perfeccionistas desadaptativos. Por eso, en este artículo se pretende realizar una revisión teórica de las investigaciones sobre el perfeccionismo para poder describir el perfil cognitivo de las personas perfeccionistas desadaptativas, 
apuntando a la detección de sus características principales. Expresamente se dejarán de lado los aspectos cognitivos del perfeccionismo saludable, ya que exceden los límites de este trabajo.

Este estudio teórico puede ser de utilidad para realizar una transferencia desde la investigación hacia la escuela, la clínica y otros ámbitos de aplicación de la psicología. Conocer el perfil cognitivo de los perfeccionistas desadaptativos puede ayudar a su detección precoz, lo que reviste gran importancia debido a las consecuencias negativas de esta problemática y su presencia en distintos ambientes, como la escuela (Schruder, Curwen \& Sharpe, 2014), la universidad (Arana et al., 2014), los deportes (Flett \& Hewitt, 2005) y la clínica médica y psicológica (Flett, Hewitt \& Molnar, 2015).

Por otra parte, las nuevas teorías incluyen al perfeccionismo entre los procesos transdiagnósticos (Egan, Wade \& Shafran, 2011), es decir que el perfeccionismo no solo es un problema en sí mismo, sino que además está presente en diversas patologías. Los procesos transdiagnósticos actúan aumentando el riesgo de desarrollar esas patologías, al tiempo que como factores mantenedores que las sostienen en el tiempo. Esto también indica la necesidad de visibilizar esta problemática en todos los entornos en los que se manifiesta y no sólo en la clínica psicológica. Además, teniendo en cuenta que los estudios científicos informan que uno de los principales problemas derivados del perfeccionismo es la disminución del efecto de la psicoterapia cuando este problema no es tratado puntualmente (Dry, Kane \& Rooney, 2015), reviste interés conocer sus componentes cognitivos para diseñar estrategias de prevención y/o de tratamiento destinadas puntualmente a ellos.

\section{El perfil cognitivo de los perfeccionistas desadaptativos}

\section{Esquemas}

Se ha descrito el desarrollo del perfeccionismo como el resultado de un interjuego entre rasgos de personalidad, en particular el neuroticismo, estilos de crianza, ambiente familiar y perfeccionismo parental (Maloney, Egan, Kane \& Rees, 2014). Y es también, a partir de las primeras experiencias en el núcleo familiar, y en relación a factores temperamentales y de personalidad, como comienzan a constituirse los esquemas maladaptativos tempranos.

Recientemente Caputto, Cordero, Keegan y Arana (2015) hallaron que el perfeccionismo desadaptativo se asociaba a todos los esquemas propuestos por Young et al. (2003), pero algunos de los dominios tenían un efecto predictivo para el perfeccionismo.

Los esquemas pertenecientes al dominio desconexión y rechazo se configuran a partir de la insatisfacción de necesidades asociadas al establecimiento de vínculos nutricios, protectores, de aceptación y apego seguro. Pertenecen a este dominio algunos esquemas característicos del 
perfeccionismo, ellos son: imperfección (i.e. la creencia de que uno es deficiente, fracasado y carece de valor), deconfianza/maltrato (i.e. la percepción de que los demás son injustos o exigen demasiado de uno, y que no son de fiar), y abandono (i.e. la sensación de que no se puede contar con los demás) (Caputto et al., 2015; Jahromi, Naziri, \& Barzegar, 2012).

En congruencia con estos hallazgos, se ha encontrado que los perfeccionistas se vinculan a partir de una base de apego inseguro (Chen et al., 2012) por lo que sienten mucha necesidad de aprobación y aceptación de los demás. Pero esa aprobación la pueden buscar implementando una estrategia de vinculación que apunta a aparentar perfección ocultando los fallos y deficiencias percibidas (i.e.autopresentación perfeccionista). Esta estrategia por lo general aumenta la desconexión, lo que confirma y mantiene los esquemas de abandono y desconfianza.

También tuvieron poder predictivo para el perfeccionismo los esquemas desadaptativos tempranos que conforman el dominio del perjuicio de autonomía y desempeño. Según la teoría, los esquemas de este dominio toman forma en un ambiente de socialización primaria sobreprotector, en el que la independencia fue coartada (Young et al., 2003). Los esquemas importantes para el perfeccionismo en este dominio son los de fracaso, vulnerabilidad al daño y dependencia/incompetencia (Caputto et al., 2015; Jahromi et al., 2012). La presencia de estos esquemas desadaptativos tempranos permite entender algunos de los componentes del perfeccionismo tales como el temor al fracaso (Conroy, Kaye, \& Fifer, 2007), la tendencia a sobreestimar y anticipar los errores (DiBartolo et al., 2007), y la necesidad de reaseguro de parte de los demás (Wu \& Wei, 2008).

El dominio de focalización en los otros también reveló potencia predictiva para el perfeccionismo desadaptativo en el estudio de Caputto et al. (2015), mientras que el dominio de sobrevigilancia e inhibición predijo el perfeccionismo en su vertiente adaptativa. Los esquemas del dominio de focalización en los otros se configuran en ambientes en los que prima la aceptación condicional (Young et al., 2003), precisamente ésta es una de las prácticas de crianza asociadas al desarrollo del perfeccionismo, en la que los padres brindan afecto a su hijo cuando cumplimenta ciertos requisitos (p.ej. sacar un diez en una evaluación escolar) y retiran el afecto cuando el niño no los cumple (Serppe et al., en prensa). En este dominio los esquemas tienen que ver con satisfacer las necesidades de los otros por sobre las propias, ya sea con la forma del esquema de autosacrificio (i.e. satisfacer voluntariamente las necesidades de los demás a expensas de las propias) o del esquema de subyugación (i.e. sentirse forzado a satisfacer a los otros). Ambos están presentes en el perfeccionismo desadaptativo, pero fundamentalmente el primero. 
Por otro lado, cuando se crece en una familia estricta, represiva y controladora se disminuyen las posibilidades de satisfacer la necesidad de espontaneidad y juego, y se desarrollan los esquemas de inhibición emocional (i.e. no desplegar las emociones para no molestar o desagradar a los demás) y de estándares inflexibles (i.e. esforzarse continuamente por lograr las metas autoimpuestas) (Young et al., 2003). El primero está más bien asociado al perfeccionismo desadaptativo y el segundo, si bien no está ausente en la versión negativa, es el componente con asociación más robusta al perfeccionismo saludable. Esto puede indicar que la búsqueda de excelencia no es negativa en sí misma, pero cuando se acompaña de otros componentes negativos puede adquirir carácter desadaptativo.

El perfeccionismo también se asocia a esquemas desadaptativos del dominio límites insuficientes, como el de autocontrol insuficiente (i.e. evitación, escaso control emocional, y tolerancia a la frustración) (Jahromi et al., 2012). Los perfeccionistas perciben que los demás no sólo les exigen en demasía sino también que son quienes tienen el control, por lo que las personas con tendencias perfeccionistas desadaptativas se sienten incapaces de lograr las metas, y aumenta su desesperanza (Periasamy \& Ashby, 2002).

Cabe señalar que el perfeccionismo desadaptativo no es el resultado lineal de la formación y persistencia de un esquema desadaptativo temprano, para llegar a ese tipo de perfeccionismo deben conjugarse otros factores. En los apartados siguientes se describirán esos factores, para continuar dando forma al perfil cognitivo del perfeccionista desadaptativo.

\section{Creencias}

El perfeccionismo ha sido definido por algunos autores como un tipo de creencia irracional en sí mismo (Ellis, 1962). Desde la perspectiva multidimensional, el deseo de perfección, y la idea de que uno será mejor, y recibirá aceptación de los demás en virtud del logro de esa anhelada perfección, es uno sus componentes cognitivos fundamentales, pero no el único. Desde este modelo, se entiende que el perfeccionismo es un constructo más amplio y complejo que excede la creencia irracional en la necesidad de ser perfecto, y que incluye también la creencia en la necesidad de que los demás sean perfectos, y la creencia de que los otros significativos demandan desempeños más que excelentes.

Las creencias relacionadas con la auto-imposición de expectativas elevadas pueden formularse en términos de "debo ser perfecto", "debo ser el mejor", "debo ganar siempre", "nunca debo equivocarme", entre otras. Albert Ellis (2002) llamó a los perfeccionistas "musturbators" (de "must", "debo" en inglés) por la tendencia a realizar demandas absolutistas por sobre las preferenciales, así una persona perfeccionista desadaptativa se impone sus metas 
en términos absolutos (p.ej. "debo ser el mejor alumno", “debo hacer las cosas siempre bien”). No es la auto-imposición de metas elevadas en sí misma lo que causa malestar a estas personas, sino la estrictez de sus propios "debería".

Es habitual que el perfeccionista desadaptativo tenga además creencias referidas a su culpabilidad y a su reactividad ante el fracaso. Esta última se refiere a una manera de catastrofizar los errores de tal modo que la persona es particularmente reactiva a las experiencias negativas (Hewitt, Flett, Blankstein \& Koledin, 1991; Flett, Hewitt \& Cheng, 2008). El deseo absoluto de hacer las cosas perfectas no se lleva bien con la reactividad al fracaso, ya que es más que esperable que alguna vez se cometa un error. Estos yerros son interpretados como el fracaso de la meta -inalcanzable- de hacer todo a la perfección, por lo que el perfeccionista se ve expuesto frecuentemente a experiencias de malestar. Sumado a esto, las creencias culpabilizantes contienen la idea de que los fallos ocurren siempre por responsabilidad propia, con lo que se desata la respuesta rumiativa y autocrítica, y aumenta el malestar.

Ahora bien, cuando el perfeccionista desadaptativo alcanza las metas que se impuso, o que percibió que le imponían, realiza una re-evaluación de sus objetivos que lo lleva a considerar ese logro como algo mínimo (p.ej. “aprobé el examen porque era muy sencillo”), y levanta sus estándares un poco más. Según se puede ver, el perfeccionista tiene dificultades para experimentar satisfacción por sus logros, ya que sus creencias lo mantienen en un círculo vicioso de exigencia y frustración (Egan et al., 2014).

Parte de las preocupaciones por el desempeño y de las reacciones ante los fallos pueden explicarse por el acuciante temor que experimentan los perfeccionistas a fracasar y a ser evaluados negativamente por los demás. El perfeccionista desadaptativo ha experimentado relaciones parentales, a partir de las cuales construyó un apego inseguro, que lo dejan en una búsqueda constante de aprobación, aceptación, y reaseguro sus logros. La situación se torna más compleja porque la persona con tendencias perfeccionistas carece de las habilidades para auto-reforzarse y autoevaluarse de una manera eficaz, que pueden ser recursos protectores y hasta mejoradores para la salud mental (Bergman, Nyland \& Burns, 2007; Chen et al., 2012; Wu \& Wei, 2008).

Nuevamente se describe un circuito de retroalimentación pero en este caso a nivel interpersonal. Los perfeccionistas se esfuerzan por ser perfectos con la expectativa de ser aceptados por los demás cuando alcancen esa perfección. Pero sus relaciones interpersonales están marcadas por la contradicción entre la necesidad de aceptación y reaseguro, el temor a ser evaluados críticamente, y la creencia en que los demás son injustos y exigentes. Los resultados 
de esas relaciones generalmente terminan siendo negativos (Hill et al., 1997), ante lo cual el perfeccionista levanta los estándares y endurece sus esfuerzos, nuevamente esperando ser aceptado cuando alcance la perfección.

Además suelen sobreestimar su responsabilidad en los eventos negativos, esta creencia en su culpabilidad frente a situaciones adversas se asocia a conductas típicas del perfeccionismo como la tendencia a la comprobación (Yorulmaz, Karanc1, \& Tekok-Kıliç, 2006). Otra creencia habitual es la idea de que es inaceptable experimentar emociones o pensamientos negativos, y que los demás evaluarán críticamente al perfeccionista si conocieran esos pensamientos o emociones (James, Verplanken \& Rimes, 2015). El perfeccionista cree que sentir malestar es un fracaso en sí mismo y esta puede ser una de las características más preocupantes del cuadro, teniendo en cuenta que es la que los lleva a sufrir en soledad.

Por último, otra de las creencias que forma parte del perfeccionismo desadaptativo es la autovaloración contingente. Las personas perfeccionistas tienden a creer que su valor personal depende de la consecución o no de las metas y logros autoimpuestos, por lo que es esperable que se consideren negativamente ya que sus fracasos son sobreestimados y los logros reevaluados y minimizados. Además, con la tendencia a anticipar el fracaso (DiBártolo et al., 2007) y con el esquema de insuficiente autocontrol, el perfeccionista está desesperanzado acerca de sus posibilidades de alcanzar el éxito y por ende, de aprobarse a sí mismo.

\section{Distorsiones cognitivas}

Estudios recientes (Yiend, Savulich,Coughtrey \& Shafran, 2011) han encontrado un sesgo en la interpretación de la realidad que es específico de los perfeccionistas, que forma parte de su perfil cognitivo y que se instala como uno de los factores mantenedores de este problema y sus trastornos asociados. Este sesgo interpretativo, que puede denominarse sesgo de perfección, se activa en circunstancias muy específicas, es decir ante estímulos que se relacionen con el contenido de las creencias y los pensamientos perfeccionistas. Esta línea de investigación es reciente y tiene algunas limitaciones, pero su potencial está en indicar que estas personas reaccionan con sesgos particulares a las situaciones en las que ven comprometidas sus metas de excelencia.

Por ejemplo, cuando un estudiante universitario con tendencias perfeccionistas desadaptativas encuentra un error ortográfico en el examen que está escribiendo, puede activarse su sesgo de perfección dando lugar al surgimiento de pensamientos relacionados con la elevación de los estándares, la anticipación de los fracasos, la autocrítica y la autovaloración contingente. Así, al ver el fallo, anticipará que desaprobará, se culpabilizará por no prestar 
suficiente atención, se impondrá el estándar de no cometer nuevamente el mismo error, reforzando la conducta perfeccionista de comprobación y llegará a pensar que, debido a su equivocación, él mismo es un fracaso. Entonces, lo que hace la interpretación de la realidad con el sesgo de perfección es, de alguna manera, gatillar el complejo de interpretaciones, emociones, pensamientos, y creencias perfeccionistas en el momento en que la persona se enfrenta a una tarea o estímulo relacionado con sus estándares o metas.

Otro elemento cognitivo que participa del procesamiento de la información de los perfeccionistas es el sesgo atencional, que implica atender selectivamente a los estímulos negativos relacionados al perfeccionismo (Howell et al., 2016; Shafran, Cooper, \& Fairburn, 2002). El sesgo atencional, o la atención selectiva, puede definirse como la atención diferencial a estímulos amenazadores en detrimento de los estímulos neutrales, y la dificultad para desengancharse de esos estímulos (Cisler, Bacon \& Williams, 2009). En el caso del estudiante universitario, por ejemplo, la atención selectiva hace que se enfoque en lo negativo (i.e. el error ortográfico) y deje de lado los aspectos positivos (i.e. el contenido del escrito que podría estar muy bien).

Junto a la atención selectiva, otro mecanismo que entra en juego es el pensamiento dicotómico. Este sesgo lleva a que la información se procese de manera polarizada, como todo o nada, y sin matices. Debido a este sesgo los perfeccionistas tienden a ver el mundo en términos de logros y fracasos; y los errores, por más pequeños que sean, entran en la categoría de fracaso (Burns \& Fedewa, 2005; Egan, Piek, Dyck \& Rees, 2007). Éste es uno de los factores que dificulta la experiencia de satisfacción respecto de los logros que pueden experimentar.

Entonces, según este procesamiento de la información, los pequeños errores son atendidos selectivamente por el perfeccionista, pasan a la categoría de fracaso e implican la no consecución de sus metas o estándares, Pero esto no quiere decir que el perfeccionista vaya a desistir en la búsqueda de la perfección. Al contrario, debido a la maximización (Bergman et al., 2007), no cesan sus esfuerzos por alcanzar las metas, se siguen esforzando y afanando hasta llegar a experimentar un malestar que probablemente silencien a razón de su creencia en que las emociones negativas son inaceptables.

Además, el perfeccionismo desadaptativo suele asociarse a la catastrofización y la sobregeneralización, de manera que los errores son magnificados al punto de convertirse en una calamidad que afecta al total de la experiencia. Se entiende así que los perfeccionistas vivencian los fallos como un fracaso, (i.e. "no sirvo para nada") y no solo como una equivocación (Davis \& Wosinski, 2012; Macedo et al., 2017). Asimismo, utilizan estándares dobles para medir sus desempeños en comparación de los demás, para quienes tienen normas más lábiles que para 
ellos mismos (Egan et al., 2014). Estas distorsiones, junto a la atención selectiva de los fallos harán que el perfeccionista esté centrado en las cosas que no salen bien, llevando el mínimo error hasta la máxima consecuencia: una tragedia que tiñe toda la producción del perfeccionista, y a su propio self, con el color del fracaso.

Si bien el sesgo atencional, el pensamiento dicotómico, la maximización, catastrofización, sobregeneralización, los debería y los estándares dobles son los más característicos del estilo de pensamiento perfeccionista, no se descarta la presencia de distorsiones tales como el etiquetado (i.e. cuando la persona se auto-asigna etiquetas que lo definan como "soy un fracasado"), el pensamiento emocional (i.e. interpretar la realidad en base a las emociones y no a los hechos, "estoy ansioso, así que seguramente desaprobaré”), la personalización (i.e. asumir la responsabilidad completa de un evento, "desaprobamos el trabajo grupal porque no me esforcé lo suficiente"), el pensamiento predictivo (vaticinar resultados, "voy a desaprobar"), y la lectura de mente (adivinar qué piensan los demás, "si desapruebo, el profesor pensará que soy un idiota”) (Egan et al., 2014).

Junto a estos sesgos y distorsiones, el perfeccionista desadaptativo posee escaso pensamiento constructivo (i.e. el tipo de pensamiento que participa de la resolución de los problemas cotidianos y que ayuda a reducir el malestar de los ajetreos diarios) (Burns \& Fedewa, 2005). Al contrario, los perfeccionistas suelen embarcarse en procesos de pensamientos negativos repetitivos que ellos mismos perciben como improductivos (Macedo et al., 2015), y que se caracterizan por perseverar, tener contenido negativo y ser sentidos como incontrolables. Estos pensamientos pueden manifestarse de forma verbal, definida, o pueden ser imágenes y hasta abstracciones imprecisas. Algunos autores sugieren que las rumiaciones y la preocupación son dos tipos de pensamiento repetitivo negativo, siendo la diferencia entre ambas el enfoque temporal que adoptan: los preocupados tienden a pensar repetitivamente sobre el futuro, mientras que los rumiadores tienden a hacerlo sobre el pasado (Ehring \& Watkins, 2008).

Las rumiaciones son un estilo de respuesta a situaciones de malestar con el que las personas, ante el estresor, tienden a enfocar la atención en sí mismas pensando una y otra vez en lo mismo. Como manera de responder al estrés es escasamente funcional, ya que la persona se enfoca en su malestar y en pensamientos asociados al mismo, pero lo hace en un estado de pasividad con el que no logra dar resolución y/o neutralizar al estresor (Nolen-Hoeksema, Wisco, \& Lyubomirsky, 2008). Otro tipo de pensamiento repetitivo negativo es la autocrítica, que puede ser entendida como el hábito mental de pensar negativamente acerca de uno mismo (Verplanken, Friborg, Wang, Trafimow \& Woolf, 2007) cuyo contenido es una respuesta 
rumiativa negativa y juiciosa de los pensamientos y emociones propios (James et al., 2015), que en el caso del perfeccionista se enfoca en los fallos y fracasos.

\section{Pensamientos Automáticos}

Flett, Hewitt, Blankstein y Gray (1998) desarrollaron el Inventario de Cogniciones Perfeccionistas (PCI) para evaluar los pensamientos automáticos de estas personas. El listado de ítems es muy claro para entender las ideas que aparecen en la conciencia de estas personas, algunas de ellas son: "debería ser perfecto", "no puedo tolerar cometer errores", "mi trabajo debe ser superior", "no importa cuánto haga, nunca es suficiente”, "¿por qué no puedo ser perfecto?", y “no debería cometer dos veces el mismo error”. Estos pensamientos automáticos pueden ser gatillados por experiencias de fracaso, que disparan la autocrítica y la autoculpabilización; pero también pueden aparecer frente a las tareas que debe completar el individuo, y llegar a interrumpir su desempeño.

Stoeber, Kobori y Tanno (2010) hicieron su aporte al validar la versión inglesa del Inventario Multidimensional de Cogniciones Perfeccionistas, en el que se leen ítems como: "mientras más alta sea la meta, mayor el desafío", "no me puedo sentir satisfecho, excepto que haga las cosas perfectas", "debo ser perfecto a cualquier costo", "me echaré la culpa si cometo un error", y "es vergonzoso cometer un error". Estos autores resaltan la idea de que evaluar estas cogniciones es importante a la hora de describir las diferencias individuales en el perfil perfeccionista de una persona.

Los pensamientos automáticos de los perfeccionistas giran en torno a algunos grandes temas: las preocupaciones acerca de ser perfecto, el afán por las metas elevadas, las auto-exigencias perfeccionistas, y las preocupaciones por los errores. Algunos autores arguyen que las personas que experimentan más pensamientos relacionados las preocupaciones por ser perfecto y por cometer errores, pueden ser más desadaptativas que aquellas cuyos pensamientos automáticos versan sobre metas elevadas y auto-exigencia (Stoeber, Kobori \& Brown, 2014). En este sentido habría pensamientos perfeccionistas negativos, positivos y neutrales o ambivalentes; verificar esta hipótesis es aún una cuenta pendiente para la investigación empírica.

En un estudio experimental, Besser, Flett, Hewitt y Guez (2008) encontraron que, ante la retroalimentación negativa posterior a una tarea (i.e. después de realizar la actividad solicitada por el experimentador, se informaba al sujeto que sus resultados estaban por debajo del promedio, independientemente de cómo haya sido su desempeño real), los individuos perfeccionistas tienden a experimentar un aumento de los pensamientos automáticos de contenido perfeccionistas, al tiempo que aumentan los pensamientos automáticos negativos 
sobre otros temas y sobre el sí mismo. Es decir que, frente a experiencias en las que los estándares no se cumplen, los perfeccionistas se ven bombardeados por una serie de pensamientos no sólo de tipo perfeccionista sino también negativos y derrotistas, que degradan su autoestima. El corolario de esto es que aumenta la discrepancia percibida entre el ideal autoimpuesto y el logro percibido y, por ende, se incrementa el malestar.

Entre esos otros pensamientos automáticos negativos que se asocian al perfeccionismo, los temas giran en torno al desajuste y deseo de cambio ("debo ser mejor"), la baja autoestima ("soy un fracasado"), y la desesperanza ("nunca lo conseguiré") (Arpin Cribbie \& Cribbie, 2007). Estos pensamientos automáticos suelen ser el contenido de las rumiaciones, es decir que perseveran en el pensamiento. Así como una golondrina no hace verano, es claro que la presencia aislada de este tipo de ideas no necesariamente es negativa; en el perfeccionismo desadaptativo la tendencia a responder con este tipo de sesgos, distorsiones y pensamientos automáticos es lo que da forma al cuadro que se describe.

\section{Afrontamiento}

La forma en que las personas se enfrentan a los estresores cotidianos o a los eventos graves de la vida, es decir el afrontamiento, está íntimamente relacionada con su estilo cognitivo. Como ya se ha dicho, los perfeccionistas desadaptativos suelen experimentar malestar en diversas maneras (p.ej. ansiedad, sentimientos depresivos, desesperanza, soledad) debido a su estilo de procesamiento de la información y otras características cognitivas. La investigación sobre los estilos de afrontamiento de los perfeccionistas ha encontrado evidencias de que el malestar que experimentan en gran medida está asociado a que los estilos que utilizan preferentemente no logran neutralizar el estrés que proviene del medio (Dunkley \& Blankstein, 2000; Dunkley, Blankstein, Halsall, Williams, \& Winkworth, 2000).

Lazarus y Folkman (1984) resaltan la importancia de atender al contexto cuando se analizan las estrategias de afrontamiento que instrumenta una persona. Esto porque, si bien es posible describir algunos estilos de afrontamiento, no es tan sencillo diferenciar los estilos adaptativos de los desadaptativos: lo que en un contexto puede ser útil para sobrellevar una situación, puede no serlo en otra. Así, una estrategia adaptativa y saludable de afrontamiento será aquella que permita que la situación estresante se resuelva y el despliegue emocional se neutralice.

La mayoría de los estudios muestran que los perfeccionistas desadaptativos suelen utilizar estrategias de afrontamiento orientadas a las emociones, en lugar de hacia la tarea (Luo, Wang, Zhang, Chen \& Quan, 2016). Las primeras son aquellas que se dirigen a regular el estado emocional, mientras que las segundas son las que apuntan a modificar el contexto, y/o la 
relación entre la persona y el contexto, generadora de malestar (Folkman, Lazarus, DunkelSchetter, DeLongis, \& Gruen, 1986). Además de la preferencia por utilizar estrategias centradas en la emoción, los perfeccionistas también son menos proclives a usar técnicas de afrontamiento enfocadas en el problema o dirigidas a buscar apoyo social (Ashby \& Gnilka, 2017).

La tendencia de los perfeccionistas a utilizar estrategias enfocadas en las emociones puede relacionarse con la creencia de que tener sentimientos negativos es inaceptable, pero más allá de eso indica que no se enfocan en cambiar la situación estresante, o resolverla. Las estrategias usadas para sobrellevar las emociones son de tipo evitativas o supresivas, y su objetivo central es dejar de sentir esas emociones. Para esto pueden utilizar la negación asociada a la paralización conductual, que no resuelve el problema e incrementa las manifestaciones emocionales (Chang, 2012).

Es decir que las técnicas supresivas o evitativas no sólo no resuelven la situación que genera malestar, sino que tampoco logran neutralizar las consecuencias emocionales. De esta manera las emociones eludidas reaparecen, la situación contextual sigue presente, el malestar no se resuelve, y el afrontamiento resulta desadaptativo (Bergman et al., 2007). Así las cosas, los perfeccionistas insanos pueden terminar reaccionando de manera impulsiva y emocional, lo que a su vez los conduce a mayores desajustes tanto psicológicos como interpersonales (Park, Hepner \& Lee, 2010).

Cuanto más intentan evitar, suprimir, y negar las emociones desagradables, más piensan en ellas. Llegan así a las rumiaciones, porque las técnicas de supresión tienden a hacer más disponibles los pensamientos que se quiere suprimir (van der Kaap-Deeder et al., 2016; Weiner \& Carton, 2012). Como estrategia de afrontamiento, la rumiación suele ser desadaptativa ya que inhabilita o paraliza la acción efectiva sobre la realidad.

El estudiante universitario que está esperando los resultados de su examen puede intentar negar su ansiedad y nerviosismo. Para esto se negará a asistir a una fiesta en la que pueda encontrarse con sus compañeros de estudio, para no ventilar sus emociones, y se quedará en su casa rumiando sus pensamientos perfeccionistas auto-culpabilizantes, autocríticos, y autoexigentes. Recordará los errores que cometió, se culpará por no haber pasado la noche en vela para repasar o, al contrario, por no haber descansado lo suficiente para tener claridad mental, y se exigirá hacerlo de una manera más estricta y organizada la próxima vez. Asociadas a esta rumiación de pensamientos perfeccionistas le surgirán ideas negativas acerca de sí mismo, de su escaso valor como estudiante y como persona, y de lo inútil que es por no poder aprobar la materia. Cae así en la trampa de la rumiación (Blankstein \& Lumnley, 2008) creyendo que al pensar en sus errores evitará volver a cometerlos, o que pensar en sus estándares 
será beneficioso para poder alcanzarlos, cuando al contrario esta rumiación improductiva le aumenta intensamente su malestar. Por otro lado, si hubiera accedido a encontrarse con sus compañeros de estudio (i.e. búsqueda de apoyo social como estrategia de afrontamiento) tal vez podría haberse beneficiado al recibir la contención y el apoyo de sus pares, y al gozar de un momento de distracción.

\section{Discusión y conclusiones}

En este trabajo se procuró describir el perfil cognitivo de los perfeccionistas desadaptativos teniendo en cuenta los elementos que generalmente lo componen: esquemas, creencias, procesamiento de la información y disfunciones cognitivas, pensamientos automáticos, y estilos de afrontamiento; con miras a hacer más asequibles los hallazgos científicos para los psicólogos, profesores y otros profesionales que se encuentran con población con tendencias perfeccionistas desadaptativas. Se seleccionaron sólo los elementos cognitivos que configuran un perfeccionismo insano o negativo, y se dejaron de lado las características saludables de la búsqueda de excelencia, ya que exceden los límites de este trabajo.

Se realizó una revisión no sistemática de la bibliografía buscando evidencias empíricas de los componentes del perfil descrito. Se utilizaron ejemplos que puedan ilustrar cómo esos componentes cognitivos se verifican en un individuo, así como también para hacer más amena la lectura.

El perfil cognitivo de los perfeccionistas desadaptativos se caracteriza por la presencia de esquemas maladaptativos tempranos, entre los que revisten particular interés aquellos que conforman el dominio de desconexión y rechazo. Estos esquemas (imperfección, desconfianza/maltrato y abandono) dan cuenta del ambiente temprano en que se desarrolló el perfeccionismo, y ponen en evidencia que la búsqueda de perfección de estas personas emerge en parte de sus ideas de ser deficientes y dañados, de la creencia de que son tratados injustamente por sus allegados que sólo le demandan desempeños ideales, y del temor a ser juzgados y abandonados si fracasan en lograr sus metas.

Ante este panorama desarrollan una serie de creencias que apuntan a la imperiosa necesidad de ser perfectos para conseguir la aprobación de los demás, pero sienten desesperanza e insuficiente autocontrol para lograr sus objetivos y con ellos, la aceptación. Sin embargo su responsabilidad ante los fracasos es grande, se sienten culpables, derrotados y sin valor, debido a la creencia de que su autovaloración es contingente a sus logros.

Sus conductas y sus mecanismos cognitivos no los ayudan para salir de la emboscada de intentar ser perfecto, al contrario procesan la información con sesgos que magnifican sus errores 
y minimizan sus aciertos. Sus conductas de comprobación, planificación y búsqueda de reaseguro surgen para disminuir el malestar y la ansiedad que se disparan cuando se activan sus pensamientos automáticos perfeccionistas, que llevan consigo a otros pensamientos negativos que perseveran de manera rumiativa, o que se convierten en un hábito mental de autocriticarse.

Como creen que es inaceptable tener pensamientos o emociones negativas, sus estrategias de afrontamiento apuntan a suprimir esas emociones más que a resolver las situaciones estresantes. Sufren en silencio, en soledad, y se enfocan en sus rumiaciones acerca de sus errores, su culpa y sus fracasos. El malestar no afloja y los perfeccionistas desadaptativos apuestan por lo que creen seguro: seguir buscando la perfección.

Esta descripción de los perfeccionistas desadaptativos no es una imagen del todo acabada, ya que el recorte elegido del perfil cognitivo deja de lado elementos tales como el afecto, las relaciones sociales y la conducta. Sin embargo, ilustra la manera en que se presenta esta problemática transdiagnóstica. Cabe recordar que, justamente en su carácter transdiagnóstico, puede hacerse visible como entidad o puede aparecer como sustrato de algún otro trastorno tal como la depresión, o los trastornos de la conducta alimentaria (Egan et al., 2011).

El perfeccionismo desadaptativo es una problemática presente en la clínica psicológica y médica, en las escuelas, las universidades y en el ámbito deportivo. Sus consecuencias negativas llegan a configurar trastornos diversos y a deteriorar la vida social de las personas, por lo que es un problema que debe ser visibilizado por fuera de los laboratorios de investigación psicológica.

Los perfiles cognitivos tienen la ventaja de ofrecer un panorama de la problemática, mostrando los elementos fundamentales para reconocerla y para intervenir en ella, ya sea de manera preventiva, terapéutica, o con fines de investigación. No se han encontrado hasta el momento artículos en los que se describa el perfil cognitivo de los perfeccionistas desadaptativos, por lo que los profesionales interesados que estén alejados de la investigación pueden tener dificultades para hallar información accesible y actualizada.

En este artículo se ha procurado llenar ese vacío, brindando una síntesis de las investigaciones sobre la temática y presentando la información de manera comprensible para profesionales de distintas áreas que estén interesados en el tema, ya sea para diseñar tratamientos ajustados o para ayudar a los consultantes a preservar su salud mental (Melgosa, 2017).

\section{Referencias}


Álvarez, N.I.T., Bedoya, V.H.C., \& Arredondo, N.H.L. (2010). Perfil cognitivo en personas con ludopatía: aproximación a la población no institucionalizada. Revista virtual universidad católica del Norte, (29), 1-24.

Arana, F.G., Galarregui, M.S., Miracco, M.C., Partarrieu, A.I., De Rosa, L., Lago, A.E., Traiber, L.I., Nusshold, P.T., Rutsztein, G. \& Keegan, E.G. (2014). Perfeccionismo y desempeño académico en estudiantes universitarios de la Ciudad Autónoma de Buenos $\begin{array}{lllll}\text { Aires. Acta } & \text { Colombiana de }\end{array}$ https://doi.org10.14718/ACP.2014.17.1.8

Arpin-Cribbie, C.A., \& Cribbie, R.A. (2007). Psychological correlates of fatigue: Examining depression, perfectionism, and automatic negative thoughts. Personality and Individual Differences, 43(6), 1310-1320. https://doi.org/10.1016/j.paid.2007.03.020.

Ashby, J. S., \& Bruner, L. P. (2005). Multidimensional perfectionism and obsessivecompulsive behaviors. Journal of College Counseling, 8(1), 31-40. http://dx.doi.org/10.1002/j.2161-1882.2005.tb00070.x

Ashby, J.S., \& Gnilka, P.B. (2017). Multidimensional perfectionism and perceived stress: Group differences and test of a coping mediation model. Personality and Individual Differences, 119, 106-111. https://doi.org/10.1016/j.paid.2017.07.012.

Bardone-Cone, A.M., Wonderlich, S.A., Frost, R.O., Bulik, C.M., Mitchell, J.E., Uppala, S., \& Simonich, H. (2007). Perfectionism and eating disorders: Current status and future directions. Clinical Psychology Review, 2007, 384-405. https://doi.org/10.1016/j.cpr.2006.12.005.

Beck, A.T., Freeman, A., Davis, D., Pretzer, J., Fleming, B, Ottaviani, R., et al. (1995). Terapia cognitiva de los trastornos de la personalidad. Buenos Aires: Paidós.

Beck, J.S. (2000). Terapia Cognitiva. Conceptos básicos y profundización. Barcelona: Gedisa. Bergman, A.J., Nyland, J.E., \& Burns, L.R. (2007). Correlates with perfectionism and the utility of a dual process model. Personality and Individual Differences, 43(2), 389-399. https://doi.org/10.1016/j.paid.2006.12.007

Besser, A., Flett, G.L., Hewitt, P.L., \& Guez, J. (2008). Perfectionism, and cognitions, affect, self-esteem, and physiological reactions in a performance situation. Journal of RationalEmotive \& Cognitive-Behavior Therapy. http://dx.doi.org/10.1007/s10942-007-0067-0.

Bieling, P. J., Israeli, A. L., \& Antony, M. M. (2004). Is perfectionism good, bad, or both? Examining models of the perfectionism construct. Personality and individual differences, 36(6), 1373-1385. https://doi.org/10.1016/S0191-8869(03)00235-6. 
Blankstein, K.R., \& Lumley, C.H. (2008). Multidimensional perfectionism and ruminative brooding in current dysphoria, anxiety, worry, and anger. Journal of Rational-Emotive \& Cognitive-Behavior Therapy, 26(3), 168-193. https://doi.org/10.1007/s10942-007-0068$\underline{\mathrm{Z}}$

Burns, L.R., \& Fedewa, B.A. (2005). Cognitive styles: Links with perfectionistic thinking. Personality and Individual Differences, 38(1), 103-113. http://dx.doi.org/10.1016/j.paid.2004.03.012.

Caputto, I., Cordero, S., Keegan, E., \& Arana, F. (2015). Perfeccionismo y esquemas desadaptativos tempranos: un estudio con estudiantes universitarios. Ciencias Psicológicas, 9(2), 245-257.

Cha, M. (2016). The mediation effect of mattering and self-esteem in the relationship between socially prescribed perfectionism and depression: Based on the social disconnection model. Personality and Individual Differences, $88 \quad$ (2016), 148-159. https://doi.org/10.1016/j.paid.2015.09.008.

Chen, C., Hewitt, P.L., Flett, G.L., Cassels, T.G., Birch, S., \& Blasberg, J.S. (2012). Insecure attachment, perfectionistic self-presentation, and social disconnection in adolescents. Personality and Individual Differences, $52 \quad$ (8), 936-941. https://doi.org/10.1016/i.paid.2012.02.009.

Cisler, J.M., Bacon, A.K., \& Williams, N.L. (2009). Phenomenological characteristics of attentional biases towards threat: A critical review. Cognitive therapy and research, 33(2), 221-234. http://dx.doi.org/10.1007/s10608-007-9161-y.

Conroy, D.E., Kaye, M.P., \& Fifer, A.M. (2007). Cognitive links between fear of failure and perfectionism. Journal of Rational-Emotive \& Cognitive-Behavior Therapy, 25(4), 237 253. https://doi.org/10.1007\%2Fs10942-007-0052-7

Davis, M.C., \& Wosinski, N.L. (2012). Cognitive errors as predictors of adaptive and maladaptive perfectionism in children. Journal of Rational-Emotive \& CognitiveBehavior Therapy, 30(2), 105-117. https://doi.org/10.1007/s10942-011-0129-1

DiBartolo, P.M., Li, C.Y., Averett, S., Skotheim, S., Smith, L.M., Raney, C., \& McMillen, C. (2007). The relationship of perfectionism to judgmental bias and psychopathology. Cognitive Therapy and Research, 31 (5), 573-587. https://doi.org/10.1007/s10608-006$\underline{9112-\mathrm{Z}}$

Dry S.M., Kane, R.T. \& Rooney, R.M. (2015). An investigation into the role of coping in preventing depression associated with perfectionism in preadolescent children. Front. Public Health 3:190. https://doi.org/10.3389/fpubh.2015.00190. 
Dunkley, D.M., \& Blankstein, K.R. (2000). Self-critical perfectionism, coping, hassles, and current distress: A structural equation modeling approach. Cognitive Therapy and Research. http://dx.doi.org/10.1023/A:1005543529245.

Dunkley, D.M., Blankstein, K.R., Halsall, J., Williams, M., \& Winkworth, G. (2000). The relation between perfectionism and distress: Hassles, coping, and perceived social support as mediators and moderators. Journal of Counseling Psychology, 47(4), 437. http://dx.doi.org/10.1037/0022-0167.47.4.437

Egan, S.J., Piek, J.P., Dyck, M.J., \& Rees, C.S. (2007). The role of dichotomous thinking and rigidity in perfectionism. Behaviour Research and Therapy, 45(8), 1813-1822. https://doi.org/10.1016/j.brat.2007.02.002.

Egan, S.J., Wade, T.D., Shafran, R., \& Antony, M.M. (2014). Cognitive-behavioural treatment of perfectionism. New York: Gilford Press.

Egan, S.J., Wade, T.D. \& Shafran, R. (2010). Perfectionism as a transdiagnostic process: A clinical review. Clinical Psychology Review, $31 \quad$ (2) 203-212. https://doi.org/10.1016/j.cpr.2010.04.009.

Ehring, T., \& Watkins, E.R. (2008). Repetitive negative thinking as a transdiagnostic process. International Journal of Cognitive Therapy, 1(3), 192-205. http://dx.doi.org/10.1680/ijct.2008.1.3.192.

Ellis, A. (1962). Reason and emotion in psychotherapy. Oxford, England: Lyle Stuart.

Ellis, A. (2002). The role of irrational beliefs in perfectionism. In G. L. Flett \& P. L. Hewitt (Eds.), Perfectionism: Theory, research, and treatment (217-229). Washington, DC, US: American Psychological Association.

Flett, G.L., Galfi-Pechenkov, I., Molnar, D.S., Hewitt, P.L., \& Goldstein, A.L. (2012). Perfectionism, mattering, and depression: A mediational analysis. Personality and Individual Differences, 52 (7), 828-832. https://doi.org/10.1016/j.paid.2011.12.041.

Flett, G.L., \& Hewitt, P.L. (2005). The perils of perfectionism in sports and exercise. Current directions in psychological science, 14(1), 14-18. https://doi.org/10.1111/j.09637214.2005.00326.x.

Flett, G.L., Hewitt, P.L., Blankstein, K.R., \& Gray, L. (1998). Psychological distress and the frequency of perfectionistic thinking. Journal of personality and social psychology, 75(5), 1363. http://dx.doi.org/10.1037/0022-3514.75.5.1363.

Flett, G.L., Hewitt, P.L., Blankstein, K.R., \& Koledin, S. (1991). Dimensions of perfectionism and irrational thinking. Journal of Rational-Emotive \& Cognitive-Behavior Therapy, 9(3), 185-201. http://dx.doi.org/10.1007/BF01061229 
Flett, G.L., Hewitt, P.L., \& Cheng, W.M.W. (2008). Perfectionism, distress, and irrational beliefs in high school students: Analyses with an abbreviated Survey of Personal Beliefs for adolescents. Journal of Rational-Emotive \& Cognitive-Behavior Therapy, 26(3), 194205. http://dx.doi.org/10.1007/s10942-007-0066-1

Flett, G.L., Hewitt, P.L., \& Molnar, D.S. (2015). Perfectionism in health and illness froma person-focused, historica perspective. En Sirois, F., \& Molnar, D. S. (Eds.) Perfectionism, health, and well-being (25-44). New York: Springer.

Flett, G.L., Nepon, T., Hewitt, P.L. \& Fitzgerald, K. (2016) Perfectionism, components of stress reactivity, and depressive symptoms. Journal of psychopathology and behavioural assessment, 38 (4) 645 - 654. http://dx.doi.org/10.1007/s10862-016-9554-X

Folkman, S., Lazarus, R.S., Dunkel-Schetter, C., DeLongis, A., \& Gruen, R.J. (1986). Dynamics of a stressful encounter: Cognitive appraisal, coping, and encounter outcomes. Journal of personality and social psychology, 50(5), 992. http://dx.doi.org/10.1037/00223514.50.5.992.

Furman, C.R., Luo, S., \& Pond, R.S. (2017). A perfect blame: Conflict-promoting attributions mediate the association between perfectionism and forgiveness in romantic relationships. Personality and Individual Differences, 111, 178-186. https://doi.org/10.1016/i.paid.2017.01.052.

Goya Arce, A.B. \& Polo, A.J. (2016). A Test of the Perfectionism Social Disconnection Model among Ethnic Minority Youth. Journal of Abnormal Child Psychology. http://dx.doi.org/10.1007/s10802-016-0240-y.

Hewitt, P.L., \& Flett, G.L. (1991). Perfectionism in the self and social contexts: conceptualization, assessment, and association with psychopathology. Journal of Personality and Social Psychology, 60 (3), 456-470. http://dx.doi.org/10.1037/00223514.60.3.456.

Hewitt, P.L. \& Flett, G.L. (1993). Dimensions of perfectionism, daily stress, and depression: a test of the specific vulnerability hypothesis. Journal of Abnormal Psychology, 102 (1), 58-65. http://dx.doi.org/10.1037/0021-843X.102.1.58.

Hewitt, P.L., Flett, G.L., Sherry, S.B., \& Caelian, C. (2006). Trait perfectionism dimensions and suicide behavior. In Ellis, T.E. (Ed) Cognition and Suicide. Theory, Research and Therapy (pp. 215-235). Washington DC. American Psychological Association.

Hill, R.W., Zrull, M.C., \& Turlington, S. (1997). Perfectionism and interpersonal problems. Journal of Personality Assessment, $69 \quad$ (1), 81-103. http://dx.doi.org/10.1207/s15327752jpa6901_5. 
Howell, J.A., McEvoy, P.M., Grafton, B., Macleod, C., Kane, R.T., Anderson, R.A., \& Egan, S.J. (2016). Selective attention in perfectionism: Dissociating valence from perfectionism-relevance. Journal of behavior therapy and experimental psychiatry, 51, 100-108. https://doi.org/10.1016/j.jbtep.2016.01.004.

Jahromi, F.G., Naziri, G., \& Barzegar, M. (2012). The relationship between socially prescribed perfectionism and depression: The mediating role of maladaptive cognitive schemas. Procedia-Social and Behavioral Sciences, 32, 141-147. https://doi.org/10.1016/j.sbspro.2012.01.023

James, K., Verplanken, B., \& Rimes, K.A. (2015). Self-criticism as a mediator in the relationship between unhealthy perfectionism and distress. Personality and Individual Differences, 79, 123-128. https://doi.org/10.1016/j.paid.2015.01.030.

Keegan, E. (2007). Escritos de psicoterapia cognitiva. Buenos Aires : Eudeba.

Lazarus, R.S., \& Folkman, S. (1984). Stress, appraisal and coping. New York; Springer.

Lazarus, R.S., \& Folkman, S. (1987). Transactional theory and research on emotions and coping. European Journal of personality, 1(3), 141-169. http://dx.doi.org/10.1002/per.2410010304.

Lemos Hoyos, M., Jaller Jaramillo, C., González Calle, A.M., Díaz León, Z.T., \& De la Ossa, D. (2012). Perfil cognitivo de la dependencia emocional en estudiantes universitarios en Medellín, Colombia. Universitas Psychologica, 11(2).

Luo, Y., Wang, Z., Zhang, H., Chen, A., \& Quan, S. (2016). The effect of perfectionism on school burnout among adolescence: The mediator of self-esteem and coping style. $\begin{array}{llll}\text { Personality } \quad \text { Individual } & \text { Differences, }\end{array}$ https://doi.org/10.1016/j.paid.2015.08.056.

Macedo, A., Marques, C., Quaresma, V., Soares, M.J., Amaral, A.P., Araújo, A.I., \& Pereira, A.T. (2017). Are perfectionism cognitions and cognitive emotion regulation strategies mediators between perfectionism and psychological distress?. Personality and Individual Differences, 119, 46-51. https://doi.org/10.1016/j.paid.2017.06.032.

Macedo, A., Soares, M.J., Amaral, A.P., Nogueira, V., Madeira, N., Roque, C., Marques, M., Maia, B., Bos, S., Valente, J. \& Pereira A.T. (2015). Repetitive negative thinking mediates the association between perfectionism and psychological distress. Personality and Individual Differences, 72, 220-224. https://doi.org/10.1016/j.paid.2014.08.024.

Maloney, G.K., Egan, S.J., Kane, R.T., \& Rees, C.S. (2014). An etiological model of perfectionism. PloS one, 9(5), e94757. https://doi.org/10.1371/journal.pone.0094757 
Magnusson, A. E., Nias, D. K. B., \& White, P. D. (1996). Is perfectionism associated with fatigue?. Journal of psychosomatic research, 41(4), 377-383. https://doi.org/10.1016/S0022-3999(96)00189-4.

Melgosa, J. (2017). Preservando la salud mental. Apuntes Universitarios, 7(2), 84-89. http://dx.doi.org/10.17162/au.v7i2.173.

Nolen-Hoeksema, S., Wisco, B.E., \& Lyubomirsky, S. (2008). Rethinking rumination. Perspectives on Psychological Science. http://dx.doi.org/10.1111/j.17456924.2008.00088.x.

Park, H.J., Heppner, P.P., \& Lee, D.G. (2010). Maladaptive coping and self-esteem as mediators between perfectionism and psychological distress. Personality and Individual Differences, 48(4), 469-474. https://doi.org/10.1016/j.paid.2009.11.024.

Pascal, E. (2003). Creencia. En Houdé, O., Kayser, D., Koening, O., Proust, J., \& Rastier, F. Diccionario de ciencias cognitivas. Neurociencia, psicología, inteligencia artificial, filosofía (117-120). Buenos Aires: Amorrortu.

Periasamy, S., \& Ashby, J.S. (2002). Multidimensional perfectionism and locus of control: Adaptive vs. maladaptive perfectionism. Journal of College Student Psychotherapy, 17(2), 75-86. http://dx.doi.org/10.1300/J035v17n02_06

Roxborough, H.M., Hewitt, P.L., Kaldas, J., Flett, G.L., Caelian, C.M., Sherry, S., \& Sherry, D.L. (2012). Perfectionistic self-presentation, socially prescribed perfectionism, and suicide in youth: A test of the perfectionism social disconnection model. Suicide and LifeThreatening Behavior, 42 (2), 217-233. $\quad$ http://dx.doi.org/10.1111/j.1943$\underline{278 X .2012 .00084 . X}$

Schruder, C.R., Curwen, T., \& Sharpe, G.W.B. (2014). Perfectionistic students: Contributing factors, impacts, and teacher strategies. British Journal of Education, Society \& Behavioural Science, 4(2), 139-155.

Serppe, M., Oros, L., Chemisquy, S., Díaz, J., Barasz, V., Waigel, N. y Ernst, C. (en prensa). ¿Qué sabemos acerca del perfeccionismo infantil en Argentina?” Revista de Psicología $U C A$

Shafran, R., Cooper, Z., \& Fairburn, C.G. (2002). Clinical perfectionism: A cognitive behavioural analysis. Behaviour Research and Therapy, 40, 773-791. https://doi.org/10.1016/S0005-7967(01)00059-6.

Sherry, S.B., Gralnick, T.M., Hewitt, P.L., Sherry, D.L., \& Flett, G.L. (2014). Perfectionism and narcissism: Testing unique relationships and gender differences. Personality and Individual Differences, 61, 52-56. https://doi.org/10.1016/j.paid.2014.01.007 
Sherry, S.B., Law, A., Hewitt, P.L., Flett, G.L. \& Besser, A. (2008). Social support as a mediator of the relationship between perfectionism and depression: A preliminary test of the social disconnection model. Personality and Individual Differences, 45 (5), 339-344. https://doi.org/10.1016/j.paid.2008.05.001.

Sirois, F.M. \& Molnar, D.M. (2016). Conceptualizations of perfectionism, health, and wellbeing: an introductory overview. En Sirois, F., \& Molnar, D. S. (Eds.). Perfectionism, health, and well-being (1-21). New York: Springer.

Stoeber, J. (2014). How other-oriented perfectionism differs from self-oriented and socially prescribed perfectionism. Journal of Psychopathology and Behavioral Assessment, 36 (2), 329-338. http://dx.doi.org/10.1007/s10862-013-9397-7

Stoeber, J. (2015). How other-oriented perfectionism differs from self-oriented perfectionism and socially prescribed perfectionism: further findings. Journal of Psychopatology and Behavioural Assessment, 37 (4), 611-623. http://dx.doi.org/10.1007/s10862-015-9485-y

Stoeber, J., Kobori, O., \& Brown, A. (2014). Examining mutual suppression effects in the assessment of perfectionism cognitions: Evidence supporting multidimensional assessment. Assessment, 21(6), 647-660. https://doi.org/10.1177/1073191114534884.

Stoeber, J., Kobori, O., \& Tanno, Y. (2010). The Multidimensional Perfectionism Cognitions Inventory-English (MPCI-E): Reliability, validity, and relationships with positive and negative affect. Journal of Personality Assessment, 92(1), 16-25. http://dx.doi.org/10.1080/00223890903379159.

Stoeber, J., \& Otto, K. (2006). Positive conceptions of perfectionism: Approaches, evidence, challenges. Personality and Social Psychology Review, 10, 295-319. http://dx.doi.org/10.1207/s15327957pspr1004_2.

van der Kaap-Deeder, J., Soenens, B., Boone, L., Vandenkerckhove, B., Stemgée, E., \& Vansteenkiste, M. (2016). Evaluative concerns perfectionism and coping with failure: Effects on rumination, avoidance, and acceptance. Personality and Individual Differences, 101, 114-119. https://doi.org/10.1016/j.paid.2016.05.063.

Vázquez, C. \& Cameron, C. (1997). Taxonomía cognitiva, psicopatología y psicoterapias cognitivas. En Caro Gabalda, I. (comp.) Manual de terapias cognitivas: estado de la cuestión y procesos terapéuticos (53-70). Barcelona: Paidós.

Verplanken, B., Friborg, O., Wang, C.E., Trafimow, D., \& Woolf, K. (2007). Mental habits: Metacognitive reflection on negative self-thinking. Journal of personality and social psychology, 92(3), 526. http://dx.doi.org/10.1037/0022-3514.92.3.526. 
Weiner, B.A., \& Carton, J.S. (2012). Avoidant coping: A mediator of maladaptive perfectionism and test anxiety. Personality and Individual Differences, 52(5), 632-636. https://doi.org/10.1016/j.paid.2011.12.009.

Wu, T.F., \& Wei, M. (2008). Perfectionism and negative mood: The mediating roles of validation from others versus self. Journal of Counseling Psychology, 55(2), 276.

Yiend, J., Savulich, G., Coughtrey, A., \& Shafran, R. (2011). Biased interpretation in perfectionism and its modification. Behaviour research and therapy, 49(12), 892-900. https://doi.org/10.1016/j.brat.2011.10.004.

Young, J.E., Klosko, J.S., \& Weishaar, M.E. (2003). Schema therapy: A practitioner's guide. Guilford Press.

Yorulmaz, O., Karanc1, A.N., \& Tekok-Kılıç, A. (2006). What are the roles of perfectionism and responsibility in checking and cleaning compulsions?. Journal of anxiety disorders, 20(3), 312-327. https://doi.org/10.1016/j.janxdis.2005.02.009

Yurica, C.L. y DiTomasso, R.A. (2005). Cognitive distortions. En A. Freeman, S.H. Felgoise, A.M. Nezu, C.M. Nezu y M.A. Reinecke (comps.), Encyclopedia of cognitive behavior therapy (117-122). Nueva York: Springer. 